\title{
Filmography as director
}

In Which We Serve, 1942, 116 mins, b/w

Production company: Two Cities Films

Directors: Noël Coward, David Lean

Producer: Noël Coward

Associate Producer: Anthony Havelock-Allan

Screenplay: Noël Coward

Adaptation [uncredited]: David Lean, Anthony Havelock-Allan, Ronald Neame

Director of Photography: Ronald Neame

Art Director: David Rawsley

Sound: C. C. Stevens

Music: Noël Coward

Editors: [David Lean], Thelma Myers

Cast: Noël Coward (Captain Kinross), Bernard Miles (Walter Hardy), John Mills (Shorty Blake), Celia Johnson (Alix Kinross), Kay Walsh (Freda Lewis), Joyce Carey (Mrs Hardy), Richard Attenborough (Stoker)

This Happy Breed, 1944, 111 mins, colour (Technicolor)

Production company: Two Cities Films

Producer: Noël Coward

Screenplay: Noël Coward, from his play

Adaptation: David Lean, Ronald Neame, Anthony Havelock-Allan

Director of Photography: Ronald Neame

Art Director: C. P. Norman

Sound: C. C. Stevens, J. C. Cook, Desmond Dew

Music Supervisor: Muir Matheson

Editor: Jack Harris

Cast: Robert Newton (Frank Gibbons), Celia Johnson (Ethel Gibbons), John Mills (Billy Mitchell), Kay Walsh (Queenie Gibbons), Stanley Holloway (Bob Mitchell) 
Blithe Spirit, 1945, 96 mins, colour (Technicolor)

Production company: Cineguild-Two Cities Films

Producer: Noël Coward

Screenplay: Noël Coward, from his play

Adaptation: David Lean, Ronald Neame, Anthony Havelock-Allan

Director of Photography: Ronald Neame

Art Director: C. P. Norman

Sound: J. C. Cook, Desmond Dew

Music: Richard Addinsell

Editor: Jack Harris

Cast: Rex Harrison (Charles Condomine), Constance Cummings (Ruth), Kay Hammond (Elvira), Margaret Rutherford (Madame Arcati), Joyce Carey (Mrs Bradman), Hugh Wakefield (Doctor Bradman), Jacqueline Clark (Edith)

\section{Brief Encounter, 1945, 86 mins, b/w}

Production company: Cineguild

Producer: Noël Coward

Screenplay: David Lean, Ronald Neame, Anthony Havelock-Allan, based on

Noël Coward's play Still Life

Adaptation: Noël Coward

Director of Photography: Robert Krasker

Art Director: L. P. Williams

Sound: Stanley Lambourne, Desmond Dew

Music: Rachmaninov's 2nd Piano Concerto played by Eileen Joyce

Editor: Jack Harris

Cast: Celia Johnson (Laura Jesson), Trevor Howard (Dr Alec Harvey), Cyril

Raymond (Fred Jesson), Joyce Carey (Myrtle Bagot), Stanley Holloway (Albert Godby), Valentine Dyall (Stephen Lynn), Everley Gregg (Dolly Messiter), Margaret Barton (Beryl)

\section{Great Expectations, 1946, 118 mins, b/w}

Production company: Cineguild

Producer: Ronald Neame

Executive Producer: Anthony Havelock-Allan

Screenplay: David Lean, Ronald Neame, Anthony Havelock-Allan with Kay

Walsh and Cecil McGivern, based on the novel by Charles Dickens

Director of Photography: Guy Green

Production Designer: John Bryan

Sound: Stanley Lambourne, Gordon K. McCallum

Music: Walter Goehr and [uncredited] Kenneth Pakeman

Editor: Jack Harris

Cast: John Mills ('Pip'), Valerie Hobson (Estella), Bernard Miles (Joe Gargery), 
Francis L. Sullivan (Jaggers), Finlay Currie (Magwitch), Martita Hunt (Miss Havisham), Anthony Wager ('Pip' as a boy), Jean Simmons (Estella as a girl), Alec Guinness (Herbert Pocket), Ivor Barnard (Wemmick), Freda Jackson (Mrs Joe Gargery), Torin Thatcher (Bentley Drummle)

Oliver Twist, 1948, 116 mins, b/w

Production company: Cineguild

Producer: Ronald Neame

Screenplay: David Lean and Stanley Haynes, from the novel by Charles Dickens

Director of Photography: Guy Green

Art Director: John Bryan

Sound: Stanley Lambourne, Gordon K. McCallum

Music: Sir Arnold Bax

Editor: Jack Harris

Cast: Robert Newton (Bill Sikes), Alec Guinness (Fagin), Fay Walsh (Nancy), John Howard Davies (Oliver), Francis L. Sullivan (Mr Bumble), Henry Stephenson (Mr Brownlow), Mary Clare (the Matron), Anthony Newley (the Artful Dodger)

The Passionate Friends, 1949, 91 mins, b/w

Production company: Cineguild

Producer: Ronald Neame

Screenplay: Eric Ambler, based on the novel by H. G. Wells

Adaptation: David Lean and Stanley Haynes

Director of Photography: Guy Green

Production Designer: John Bryan

Sound: Stanley Lambourne, Gordon K. McCallum

Music: Richard Addinsell

Editor: Jack Harris

Cast: Ann Todd (Mary Austin), Trevor Howard (Steven Stratton), Claude Rains (Howard Justin)

Madeleine, 1950, 114 mins, b/w

Production company: Cineguild

Producer: Stanley Haynes

Screenplay: Stanley Haynes and Nicholas Phipps

Director of Photography: Guy Green

Art Director: John Bryan

Sound: Gordon K. McCallum

Music: William Alwyn

Editor: Geoffrey Foot 
Cast: Ann Todd (Madeleine Smith), Ivan Desny (Emile L'Angelier), Norman Woland (William Minnoch), Leslie Banks (Mr Smith), Barbara Everest (Mrs Smith), Ivor Barnard (Mr Murdoch)

The Sound Barrier, 1952, 118 mins, b/w

Production company: London Films

Producer: David Lean

Associate Producer: Norman Spencer

Screenplay: Terence Rattigan

Director of Photography: Jack Hildyard

Production Design: Vincent Korda

Sound: John Cox

Music: Malcolm Arnold

Editor: Geoffrey Foot

Cast: Ralph Richardson (Sir John Ridgefield), Ann Todd (Susan Ridgefield

Garthwaite), Nigel Patrick (Tony Garthwaite), John Justin (Philip Peel),

Dinah Sheridan (Jess Peel), Joseph Tomelty (Will Sparks), Denholm Elliott (Chris Ridgefield)

Hobson's Choice, 1954, 107 mins, b/w

Production company: London Films in Association with British Lion

Producer: David Lean

Associate Producer: Norman Spencer

Screenplay: David Lean, Norman Spencer and Wynyard Browne, based on the play by Harold Brighouse

Director of Photography: Jack Hildyard

Art Directed by Wilfred Shingleton

Sound: John Cox

Music: Malcolm Arnold

Cast: Charles Laughton (Henry Hobson), Brenda de Banzie (Maggie), John Mills (Will Mossop), Daphne Anderson (Alice Hobson), Prunella Scales (Vicky Hobson), Richard Wattis (Albert Prosser)

\section{Summer Madness, 1955, 99 mins, colour (Eastmancolor)}

Production company: Lopert Films/London Films

Producer: Ilya Lopert

Associate Producer: Norman Spencer

Screenplay: David Lean, H. E. Bates, based on the play Time of the Cuckoo by Arthur Laurents

Director of Photography: Jack Hildyard

Art Director: Vincent Korda

Sound: Peter Handford 
Music: Alessandro Cicognini; La gazza ladra by Giacomo Rossini

Editor: Peter Taylor

Cast: Katharine Hepburn (Jane Hudson), Rossano Brazzi (Renato Di Rossi), Isa Miranda (Signora Fiorina), Darren McGavin (Eddie Jaeger), Mari Aldon (Phyl Jaeger), Jane Rose (Edith McIlhenny), MacDonald Parke (Lloyd McIlhenny), Gaetano Autiero (Mauro)

\section{The Bridge on the River Kwai, 1957, 160 mins, colour (Technicolor)}

Production company: Horizon Pictures

Producer: Sam Spiegel

Screenplay: Pierre Boulle and [uncredited] Carl Foreman, Calder Willingham, Michael Wilson, and David Lean, based on Boulle's novel. Due to blacklisting, neither Foreman nor Wilson received script credit, which was given to author Boulle, who had nothing to do with scripting the film.

Director of Photography: Jack Hildyard

Art Director: Donald M. Ashton

Sound: John Cox, John Mitchell

Music: Malcolm Arnold; 'Colonel Bogey March' by Kenneth J. Alford

Editor: Peter Taylor

Cast: Alec Guinness (Colonel Nicholson), William Holden (Shears), Jack Hawkins (Major Warden), Sessue Hayakawa (Colonel Saito), James Donald (Dr Clipton), Geoffrey Horne (Lieutenant Joyce), Andre Morell (Colonel Green), Percy Herbert (Grogan)

Lawrence of Arabia, 1962, 207 mins, colour (Technicolor)

Production company: Horizon Films

Producer: Sam Spiegel

Screenplay: Robert Bolt (draft screenplay by Michael Wilson)

Director of Photography: Freddie Young

Production Designer: John Box

Sound: Paddy Cunningham

Music: Maurice Jarre; 'The Voice of the Guns' by Kenneth J. Alford

Editor: Anne V. Coates

Cast: Peter O'Toole (Thomas Edward Lawrence), Alec Guinness (Prince Feisal), Anthony Quinn (Auda), Jack Hawkins (General Allenby), Omar Sharif (Sherif Ali), Anthony Quayle (Colonel Brighton), Claude Rains (Mr Dryden), Arthur Kennedy (Jackson Bentley), José Ferrer (Turkish Bey), Donald Wolfit (General Murray) 
Doctor Zhivago, 1965, 193 mins, colour (Metrocolor)

Production company: Carlo Ponti/MGM

Producer: Carlo Ponti

Executive Producer: Arvid L. Griffen

Screenplay: Robert Bolt, based on the novel by Boris Pasternak

Director of Photography: Freddie Young

Production Designer: John Box

Sound: Paddy Cunningham

Music: Maurice Jarre

Editor: Norman Savage

Cast: Omar Sharif (Yuri Zhivago), Julie Christie (Lara), Geraldine Chaplin (Tonya Gromeko Zhivago), Tom Courtenay (Pasha/Strelnikov), Alec Guinness (General Yegraf Zhivago), Siobhan McKenna (Anna Gromeko), Ralph Richardson (Alexander Gromeko), Rod Steiger (Komarovsky), Rita Tushingham (Tonya), Klaus Kinski (Kostoyed)

\section{Ryan's Daughter, 1970, 206 mins, colour (Metrocolor)}

Production company: Faraway Productions/MGM

Producer: Anthony Havelock-Allan

Associate Producer: Roy Stevens

Screenplay: Robert Bolt

Director of Photography: Freddie Young

Production Designer: Stephen Grimes

Sound: John Bramwell

Music: Maurice Jarre

Editor: Norman Savage

Cast: Sarah Miles (Rosy Ryan), Robert Mitchum (Charles Shaughnessy), Trevor Howard (Father Hugh Collins), Christopher Jones (Major Randolph Doryan), John Mills (Michael), Leo McKern (Tom Ryan), Barry Foster (Tim O'Leary)

Lost and Found: The Story of Cook's Anchor (TV), 1979, 40 mins, colour

Production company: South Pacific TV/Faraway Productions

Producers: George Andrews, Wayne Tourell

Teleplay: Robert Bolt, David Lean, Wayne Tourell

Photography: Ken Dorman, Lynton Diggle, Eddie Fowlie

Editor: David Reed 


\section{FILMOGRAPHY AS DIRECTOR}

\section{A Passage to India, 1984, 163 mins, colour (Technicolor)}

Production company: G. W. Films, John Heyman, Edward Sands, Home Box Office

Producers: John Brabourne, Richard B. Goodwin

Executive Producers: John Heyman, Edward Sands

Screenplay: David Lean, based on the novel by E. M. Forster and the play by Santha Rama Rau

Director of Photography: Ernest Day

Production Designer: John Box

Sound: Graham V. Hartstone, Nicolas Le Messurier, Michael A. Carter and John W. Mitchell

Music: Maurice Jarre; 'Freely Maisie' by John Dalby

Editor: David Lean

Cast: Judy Davis (Adela Quested), Victor Banerjee (Dr Aziz), Peggy Ashcroft (Mrs Moore), James Fox (Fielding), Alec Guinness (Godbole), Nigel Havers (Ronny Heaslop), Richard Wilson (Turton), Michael Culver (McBryde), Art Malik (Mahmoud Ali), Saeed Jaffrey (Hamidullah) 